Editorials

\title{
Fuzzy Bernstein-Stancu Operator of Rough I-Core of Triple Sequences
}

\author{
${ }^{1}$ Ayhan Esi, ${ }^{2}$ Nagarajan Subramanian and ${ }^{3}$ M. Kemal Ozdemir \\ ${ }^{1}$ Department of Mathematics, Adiyaman University, Adiyaman, 02040, Turkey \\ ${ }^{2}$ Department of Mathematics, School of Arts Sciences and Humanities, \\ SASTRA Deemed to be University, Thanjavur-613 401, India \\ ${ }^{3}$ Department of Mathematics, Inonu University, 44280, Malatya, Turkey
}

\author{
Article history \\ Received: 16-09-2019 \\ Revised: 16-10-2019 \\ Accepted: 16-12-2019 \\ Corresponding Author: \\ Ayhan Esi \\ Department of Mathematics, \\ Adiyaman University, \\ Adiyaman, 02040, Turkey \\ Email: aesi23@hotmail.com
}

\begin{abstract}
We are going to present and investigate some fundamental characteristics of fuzzy Bernstein-Stancu polynomials of rough Iconvergent of triple sequence spaces and at the same time examine the set of all fuzzy Bernstein Stancu polynomials of rough I-limits of a triple sequence spaces and also relation between analyticness and fuzzy Bernstein Stancu polynomials of rough I-core of a triple sequence spaces.
\end{abstract}

Keywords: Ideal, Triple Sequences, Rough Convergence, Closed and Convex, Cluster Points and Rough Limit Points, Bernstein Stancu Operator, Fuzzy Numbers, Fuzzy Sets

\section{Introduction}

The idea of rough convergence was first introduced by Phu $(2001 ; 2002 ; 2003)$ in dimensional normed spaces which is finite. He showed that the set $\operatorname{LIM}_{x}^{r}$ is bounded, closed and convex; and he introduced the notion of rough Cauchy sequence. He also investigated the relations between rough convergence and other convergence kinds and the dependence of $\operatorname{LIM}_{x}^{r}$ on the roughness of degree denoted by $r$.

Aytar (2008a) studied of rough statistical convergence and described the rough statistical set's limit points of a given sequence and obtained two statistical convergence criteria associated with this set and prove that the set is convex and closed. Also, Aytar (2008b) studied that the fact that $r$-limit set of the sequence is equal to intersection of these sets and that $r$ core of the sequence is equal to the union of these sets. Dundar and Cakan (2014) have studied of rough ideal convergence and described the rough ideal limit set's points of a sequence. The notion of $I$-convergence for a triple sequence spaces which is based on the very structure of the ideal $I$ of subsets of $\mathbb{N}^{3}$, where $\mathbb{N}$ is the set of all natural numbers, is a natural generalization of the notion of convergence and statistical convergence.

In this paper we are going to examine some basic properties of rough I-convergence of a triple sequence spaces of fuzzy in three dimensional matrix spaces which are not earlier. We study the set of all rough $I$ limits of a triple sequence spaces of fuzzy and also the relation between analyticness and rough $I$-core of a triple sequence spaces of fuzzy. Let us assume that $K$ be a subset of the set of positive integers $\mathbb{N}^{3}$ and let us denote the set $K_{i j l}=\{(m, n, k) \in K: m \leq i, n \leq j, k \leq \ell\}$. Then the natural density of $K$ is given by:

$$
\delta_{3}(K)=\lim _{i, j, \ell \rightarrow \infty} \frac{\left|K_{i j \ell}\right|}{i j \ell},
$$

where, $\left|K_{i j \ell}\right|$ denotes the number of elements in $K_{i j \ell}$.

First of all, we have applied the concept of $(p, q)$ calculus in approximate theory and introduced the $(p, q)$ analogue of Bernstein operators. Next, based on $(p, q)$ integers, some approximate results for Bernstein-Stancu operators, Bernstein-Kantorovich operators, $(p, q)$ Lorentz operators, Bleimann-Butzer and Hahn operators and Bernstein-Shurer operators etc.

Khan and Lobiyal (2015) gave a very nice application in computer-aided geometric design and used these Bernstein basis for construction of $(p, q)$-Bezier curves and surfaces based on $(p, q)$-integers which is further generalization of $q$-Bezier curves and surfaces.

Being motivated with the above described study on $(p, q)$-approximation and its application, in the present paper we are going to investigate statistical approximate characteristics of Bernstein-Stancu operators based on $(p, q)$-integers.

Now we recall some basic definitions about $(p, q)$ integers. For any given $u, v, w \in \mathbb{N}$, the (p; q)-integer $[u v w]_{p, q}$ is defined by: 


$$
[0]_{p, q}:=0 \text { and }[u v w]_{p, q}=\frac{p^{u v w}-q^{u v w}}{p-q} \text { if } u, v, w \geq 1,
$$

where, $0<q<p \leq 1$. Here the $(p, q)$-factorial is defined by:

$$
[0]_{p, q} !:=1 \text { and }[u v w] !_{p, q}=[1]_{p, q}[2]_{p, q} \ldots[u v w]_{p, q} \text { if } u, v, w \geq 1 \text {. }
$$

Also the $(p, q)$-binomial coefficient is defined by:

$$
\begin{aligned}
& \left(\begin{array}{c}
u \\
m
\end{array}\right)\left(\begin{array}{l}
v \\
n
\end{array}\right)\left(\begin{array}{c}
w \\
k
\end{array}\right)_{p, q} \\
& =\frac{[u] !_{p, q}}{[m] !_{p, q}[u-m] !_{p, q}} \frac{[v] !_{p, q}}{[n] !_{p, q}[v-n] !_{p, q}} \frac{[w] !_{p, q}}{[k] !_{p, q}[w-k] !_{p, q}}
\end{aligned}
$$

for every $u, v, w, m, n, k \in \mathbb{N}$ having $u \geq m, v \geq n, w \geq k$.

The formula for $(p, q)$-binomial expansion is as follows:

$$
\begin{aligned}
& (a x+b y)_{p, q}^{u v w} \\
& =\sum_{m=0}^{u} \sum_{n=0}^{v} \sum_{k=0}^{w} p^{\frac{(u-m)(u-m-1)+(v-n)(v-n-1)+(w-k)(w-k-1)}{2}} q^{\frac{m(m-1)+n((n-1)+k(k-1)}{2}} \\
& \left(\begin{array}{c}
u \\
m
\end{array}\right)\left(\begin{array}{c}
v \\
n
\end{array}\right)\left(\begin{array}{c}
w \\
k
\end{array}\right)_{p, q} a^{(u-m)+(v-n)+(w-k)} b^{m+n+k} x^{(u-m)+(v-n)+(w-k)} y^{m+n+k}, \\
& (x+y)_{p, q}^{u w w}=(x+y)(p x+q y)\left(p^{2} x+q^{2} y\right) \cdots \\
& \left(p^{(u-1)+(v-1)+(w-1)} x+q^{(u-1)+(v-1)+(w-1)} y\right), \\
& (1-x)_{p, q}^{u w w}=(1-x)(p-q x)\left(p^{2}-q^{2} x\right) \cdots \\
& \left(p^{(u-1)+(v-1)+(w-1)}-q^{(u-1)+(v-1)+(w-1)} x\right),
\end{aligned}
$$

and:

$$
(x)_{p, q}^{m m k}=x(p x)\left(p^{2} x\right) \cdots\left(p^{(u-1)+(v-1)+(w-1)} x\right)=p^{\frac{m(m-1)+n(n-1)+k(k-1)}{2}} .
$$

The Bernstein operator having order $r s t$ is given by:

$$
\begin{aligned}
& B_{r s t}(f, x) \\
& =\sum_{m=0}^{r} \sum_{n=0}^{s} \sum_{k=0}^{t} f\left(\frac{m n k}{r s t}\right)\left(\begin{array}{l}
r \\
m
\end{array}\right)\left(\begin{array}{l}
s \\
n
\end{array}\right)\left(\begin{array}{l}
t \\
k
\end{array}\right) x^{m+n+k}(1-x)^{(m-r)+(n-s)+(k-t)}
\end{aligned}
$$

where $f$ is a given continuous function (which may be real or complex valued one) function defined on the interval $[0,1]$.

$(p, q)$-Bernstein operators are defined as follows:

$$
\begin{aligned}
& B_{r s t, p, q}(f, x) \\
& =\frac{1}{p} \frac{1(r-1)+(s-1)+(t(-1)}{2} \sum_{m=0}^{r} \sum_{n=0}^{s} \sum_{k=0}^{t}\left(\begin{array}{l}
r \\
m
\end{array}\right)\left(\begin{array}{l}
s \\
n
\end{array}\right)\left(\begin{array}{l}
t \\
k
\end{array}\right) p^{\frac{m(m-1)+n(n-1)+k(k-1)}{2}} x^{m+n+k} . \\
& \prod_{u_{1}=0}^{(r-m-1)}\left(p^{u_{1}}-q^{u_{1}} x\right) \prod_{u_{2}=0}^{(s-n-1)}\left(p^{u_{2}}-q^{u_{2}} x\right) \prod_{u_{3}=0}^{(t-k-1)}\left(p^{u_{3}}-q^{u_{3}} x\right) . \\
& f\left(\frac{p^{(r-m)}[m]_{p, q}+p^{(s-n)}[n]_{p, q}+p^{(t-k)}[k]_{p, q}}{[r]_{p, q}+[s]_{p, q}+[t]_{p, q}}\right), x \in[0,1]
\end{aligned}
$$

Also, we have:

$$
\begin{aligned}
& (1-x)_{p, q}^{r s t} \\
& =\sum_{m=0}^{r} \sum_{n=0}^{s} \sum_{k=0}^{t}(-1)^{m+n+k} p \frac{(r-m)(r-m-1)+(s-n)(s-n-1)+(t-k)(t-k-1)}{2} q \frac{m(m-1)+n((n-1)+k(k-1)}{2} . \\
& \left(\begin{array}{c}
r \\
m
\end{array}\right)\left(\begin{array}{c}
s \\
n
\end{array}\right)\left(\begin{array}{c}
t \\
k
\end{array}\right) x^{m+n+k} . \\
& S_{r s t, p, q}(f, x) \\
& =\frac{1}{p} \frac{r(r-1)+s(s-1)+t(t-1)}{2} \sum_{m=0}^{r} \sum_{n=0}^{s} \sum_{k=0}^{t}\left(\begin{array}{l}
r \\
m
\end{array}\right)\left(\begin{array}{l}
s \\
n
\end{array}\right)\left(\begin{array}{l}
t \\
k
\end{array}\right) p^{\frac{m(m-1)+n(n-1)+k(k-1)}{2}} x^{m+n+k} . \\
& \prod_{u_{1}=0}^{(r-m-1)}\left(p^{u_{1}}-q^{u_{1}} x\right) \prod_{u_{2}=0}^{(s-n-1)}\left(p^{u_{2}}-q^{u_{2}} x\right) \prod_{u_{3}=0}^{(t-k-1)}\left(p^{u_{3}}-q^{u_{3}} x\right) . \\
& f\left(\frac{p^{(r-m)}[m]_{p, q}+p^{(s-n)}[n]_{p, q}+p^{(t-k)}[k]_{p, q}+\eta}{[r]_{p, q}+[s]_{p, q}+[t]_{p, q}+\mu}\right), x \in[0,1]
\end{aligned}
$$

Let us note the fact that for $\eta=\mu=0,(p, q)$ Bernstein-Stancu operators given by (1.2) converts into $(p, q)$-Bernstein operators. At the same time for $p=1,(p$, $q$ )-Bernstein-Stancu operators given by (1.1) turn out to be q-Bernstein-Stancu operators.

Throughout the paper, $\mathbb{R}$ denotes the real of three dimensional space with metric $(X, d)$. Let us consider a triple sequence of Bernstein-Stancu polynomials $\left(S_{r s t, p, q}\right.$ $(f, x))$ such that $\left(S_{r s t, p, q}(f, x)\right) \in \mathbb{R}, m, n, k \in \mathbb{N}$.

Let $f$ be a given continuous function defined over the closed range interval $[0,1]$. A triple sequence of Bernstein-Stancu polynomials $\left(S_{r s t, p, q}(f, x)\right)$ is said to be statistically convergent to $(f, x)$, written as $s t_{3}-\lim S_{r s t, p, q}$ $(f, x)=f(x)$, provided that

the set:

$$
K_{\epsilon}:=\left\{(m, n, k) \in \mathbb{N}^{3}:\left|S_{r s t, p, q}(f, x)-(f, x)\right| \geq \epsilon\right\}
$$

has natural density zero for any $\epsilon>0$. In this case, $(f, x)$ is called the statistical limit of the triple sequence of Bernstein-Stancu polynomials. i.e., $\delta_{3}\left(K_{\epsilon}\right)=0$. That is: 


$$
\lim _{r, s, t \rightarrow \infty} \frac{1}{r s t}\left|\left\{m \leq r, n \leq s, k \leq t:\left|S_{r s t, p, q}(f, x)-(f, x)\right| \geq \epsilon\right\}\right|=0 .
$$

In this case, we write $\delta_{3}$-lim $S_{r s t, p, q}(f, x)=(f, x)$ or $S_{r s t, p, q}(f, x) \stackrel{s t 3}{\longrightarrow}(f, x)$.

Throughout the paper, $\mathbb{N}$ denotes the set of all positive integers, $\chi_{A^{-}}$the characteristic function of $A \subset \mathbb{N}, \mathbb{R}$ the set of all real numbers. A subset $A$ of $\mathbb{N}^{3}$ is said to have asymptotic density $d_{3}(A)$ if:

$$
d_{3}(A)=\lim _{i, j, \ell \rightarrow \infty} \frac{1}{i j \ell} \sum_{m=1}^{i} \sum_{n=1}^{j} \sum_{k=1}^{\ell} \chi_{A}(K) .
$$

The theory of statistical convergence has been discussed in trigonometric series, summability theory, measure theory, turnpike theory, approximation theory, fuzzy set theory and so on.

A triple sequence (real or complex) can be defined as a function $x: \mathbb{N} \times \mathbb{N} \times \mathbb{N} \rightarrow \mathbb{R}(\mathbb{C})$, where $\mathbb{N}, \mathbb{R}$ and $\mathbb{C}$ denote the set of natural numbers, real numbers and complex numbers, respectively. The different types of notions of triple sequence was introduced and investigated at the initial by Sahiner et al. (2007; Sahiner and Tripathy, 2008), Esi (2014; Esi and Catalbas, 2014; Esi and Savas, 2015; Esi et al., 2016), Dutta et al. (2013), Subramanian and Esi (2015), Debnath et al. (2015) and many others.

A triple sequence $x=\left(x_{m n k}\right)$ is said to be triple analytic if:

$$
\sup _{m, n, k}\left|x_{m n k}\right|^{\frac{1}{m+n+k}}<\infty .
$$

The space of all triple analytic sequences are usually denoted by $\Lambda^{3}$.

A fuzzy number $X$ is a fuzzy subset of the real $\mathbb{R}^{3}$, which is normal fuzzy convex, upper semi-continuous and the $X^{0}$ is bounded where $X^{0}:=\operatorname{cl}\left\{x \in \mathbb{R}^{3}: X(x)>0\right\}$ and $\mathrm{cl}$ is the closure operator. These properties imply that for each $\alpha \in(0,1]$, the $\alpha$-level set $X^{\alpha}$ defined by:

$$
X^{\alpha}=\left\{x \in \mathbb{R}^{3}: X(x) \geq \alpha\right\}=\left[\underline{X}^{\alpha}, \bar{X}^{\alpha}\right]
$$

is a non empty compact convex subset of $\mathbb{R}^{3}$.

The supremum metric d on the set $L\left(\mathbb{R}^{3}\right)$ is defined by:

$$
d(X, Y)=\sup _{\alpha \in[0,1]} \max \left(\left|\underline{X}^{\alpha}-\underline{Y}^{\alpha}\right|-\left|\bar{X}^{\alpha}-\bar{Y}^{\alpha}\right|\right) .
$$

Now, given $X, Y \in L\left(\mathbb{R}^{3}\right)$, we define $X \leq Y$ if $\underline{X}^{\alpha} \leq \underline{Y}^{\alpha}$ and $\bar{X}^{\alpha} \leq \bar{Y}^{\alpha}$ for each $\alpha \in[0,1]$.
We write $X \leq Y$ if $X \leq Y$ and there exists an $\alpha_{0} \in[0,1]$ such that $\underline{X}^{\alpha_{0}} \leq \underline{Y}^{\alpha_{0}}$ or $\bar{X}^{\alpha_{0}} \leq \bar{Y}^{\alpha_{0}}$.

A subset $E$ of $L\left(\mathbb{R}^{3}\right)$ is said to be bounded above if there exists a fuzzy number $\mu$, called an upper bound of $E$, such that $X \leq \mu$ for every $X \in E$. $\mu$ is called the least upper bound of $E$ if $\mu$ is an upper bound and $\mu \leq \mu^{\prime}$ for all upper bounds $\mu^{\prime}$.

A lower bound and the greatest lower bound are defined similarly. $E$ is said to be bounded if it is both bounded above and below.

The notions of least upper bound and the greatest lower bound have been defined only for bounded sets of fuzzy numbers. If the set $E \subset L\left(\mathbb{R}^{3}\right)$ is bounded then its supremum and infimum exist.

The limit infimum and limit supremum of a triple sequence spaces $\left(X_{m n k}\right)$ is defined by:

$$
\begin{aligned}
& \lim _{m n k \rightarrow \ell} \inf X_{m n k}:=\inf A_{X} . \\
& \lim _{m n k \rightarrow \ell} \sup X_{m n k}:=\inf B_{X} .
\end{aligned}
$$

where:

$$
\begin{aligned}
& A_{X}:=\left\{\mu \in L\left(\mathbb{R}^{3}\right): \text { The } \operatorname{set}\left\{(m, n, k) \in \mathbb{N}^{3}: X_{m n k}<\mu\right\} \text { is infinite }\right\} \\
& B_{X}:=\left\{\mu \in L\left(\mathbb{R}^{3}\right): \text { The set }\left\{(m, n, k) \in \mathbb{N}^{3}: X_{m n k}<\mu\right\} \text { isinfinite }\right\} .
\end{aligned}
$$

Now, given two fuzzy numbers $X, Y \in L\left(\mathbb{R}^{3}\right)$, we define their sum as $Z=X+Y$, where $\underline{Z}^{\alpha}:=\underline{X}^{\alpha}+\underline{Y}^{\alpha}$ and $\bar{Z}^{\alpha}:=\bar{X}^{\alpha}+\bar{Y}^{\alpha}$ for all $\alpha \in[0,1]$.

To any real number $a \in \mathbb{R}^{3}$, we can assign a fuzzy number $a_{1} \in L\left(\mathbb{R}^{3}\right)$, which is defied by:

$$
a_{1}(x)=\left\{\begin{array}{l}
1, \text { if } x=\alpha, \\
0, \text { otherwise }
\end{array} .\right.
$$

An order interval in $L\left(\mathbb{R}^{3}\right)$ is defined by $[X, Y]:=$ $\left\{Z \in L\left(\mathbb{R}^{3}\right): X \leq Z \leq Y\right\}$, where $X, Y \in L\left(\mathbb{R}^{3}\right)$.

A set $E$ of fuzzy numbers is called convex if $\lambda \mu_{1}+$ $(1-\lambda) \mu_{2} \in E$ for all $\lambda \in[0,1]$ and $\mu_{1}, \mu_{2} \in E$.

\section{Definitions and Preliminaries}

Throughout the paper $\mathbb{R}^{3}$ denotes the real three dimensional case with the metric. Consider a triple sequence $x=\left(x_{m n k}\right)$ such that $x_{m n k} \in \mathbb{R}, m, n, k \in \mathbb{N}$. Those definitions follows:

\section{Definition 2.1}

Let $f$ be a given continuous function defined over the given closed interval $[0,1]$. A triple sequence of fuzzy 
Bernstein-Stancu polynomials $\left(S_{r s t, p, q}(f, X)\right)$ is said to be statistically convergent to $(f, X)$ given by $S_{r s t, p, q}(f, X) \rightarrow^{s t-\lim x}$ $(f, X)$, if for every $\epsilon>0$ we have $d(A(\epsilon))=0$, where:

$$
A(\epsilon)=\left\{(r, s, t) \in \mathbb{N}^{3}:\left|S_{r s t, p, q}(f, X)-(f, X)\right| \geq \epsilon\right\} .
$$

\section{Definition 2.2}

Let $f$ be a given continuous function defined over the closed interval $[0,1]$. A triple sequence of fuzzy Bernstein-Stancu polynomials $\left(S_{r s t, p, q}(f, X)\right)$ is said to be statistically convergent to $(f, X)$ symbolized by $S_{r s t, p, q}(f$, $X) \rightarrow^{s t-\lim x}(f, X)$, provided that the set:

$$
\left\{(r, s, t) \in \mathbb{N}^{3}:\left|S_{r s t, p, q}(f, X)-(f, X)\right| \geq \epsilon\right\},
$$

has natural density zero for every $\epsilon>0$. In this case, $(f$, $X$ ) is called the statistical limit of the sequence of fuzzy Bernstein-Stancu polynomials.

\section{Definition 2.3}

Let $f$ be a given continuous function defined over the closed interval $[0,1]$. A triple sequence of fuzzy Bernstein-Stancu polynomials $\left(S_{r s t, p, q}(f, X)\right)$ in a metric space $(X, \mid .,$.$) and \beta$ be a non-negative real number is said to be $\beta$-convergent to $(f, X)$, symbolized by $S_{r s t, p, q}(f$, $X) \rightarrow^{\beta}(f, X)$, if for any $\epsilon>0$ there exists $N_{\epsilon} \in \mathbb{N}$ such that for all $r, s, t \geq N_{\epsilon}$ we have:

$$
\left|S_{r s t, p, q}(f, X)-(f, X)\right|<r+\epsilon
$$

Under these conditions $S_{r s t, p, q}(f, X)$ is known as an $\beta$ limit of $(f, X)$.

\section{Remark 2.1}

We are going to consider $\beta$-limit set $S_{r s t, p, q}(f, X)$ which is described by $\operatorname{LIM}^{\beta} S_{r s t, p, q}(f, X)$ and is defined by:

$$
\operatorname{LIM}^{\beta} S_{r s t, p, q}(f, X)=\left\{f: S_{r s t, p, q}(f, X) \rightarrow^{\beta}(f, X)\right\} .
$$

\section{Definition 2.4}

Let $f$ be a continuous function defined on the closed interval $[0,1]$.

A triple sequence of fuzzy Bernstein-Stancu polynomials $\left(S_{r s t, p, q}(f, X)\right)$ is said to be $\beta$-convergent if $\operatorname{LIM}^{\beta} S_{r s t, p, q}(f, X) \neq \phi$ and $\beta$ is called a rough convergence degree of $S_{r s t, p, q}(f, X)$. If $\beta=0$ then it is ordinary convergence of triple sequence of fuzzy Bernstein-Stancu polynomials.

\section{Definition 2.5}

Let $f$ be a given continuous function defined over the closed interval $[0,1]$. A triple sequence of fuzzy
Bernstein-Stancu polynomials $\left(S_{r s t, p, q}(f, X)\right)$ in a metric space $(X, \mid .,$.$) and \beta$ be a non-negative real number is said to be $\beta$-statistically convergent to $(f, X)$, denoted by $S_{r s t, p, q}(f, X) \rightarrow^{\beta-s t_{3}}(f, X)$, if for any $\epsilon>0$ we conclude that $d_{3}(A(\epsilon))=0$, where:

$$
A(\epsilon)=\left\{(r, s, t) \in \mathbb{N}^{3}:\left|S_{r s t, p, q}(f, X)-(f, X)-(f, X)\right| \geq r+\epsilon\right\} .
$$

In this case $(f, X)$ is called $\beta$-statistical limit of $S_{r s t, p, q}$ $(f, X)$. If $\beta=0$ then it is ordinary statistical convergent of triple sequence of fuzzy Bernstein-Stancu polynomials.

\section{Definition 2.6}

The class denoted by $I$ of subsets of a given nonempty set $X$ is said to be an ideal in $X$ provided:

(i) $\phi \in I$;

(ii) $A, B \in I$ implies $A \cup B \in I$

(iii) $A \in I, B \subset A$ implies $B \in I$

$I$ is called a nontrivial ideal if $X \notin I$.

\section{Definition 2.7}

A nonempty class $F$ of subsets of a nonempty set $X$ is said to be a filter in $X$. Provided:

(i) $\phi \in F$

(ii) $A, B \in F$ implies $A \cap B \in F$;

(iii) $A \in F, A \subset B$ implies $B \in F$.

\section{Definition 2.8}

$I$ is a non trivial ideal in $X, X \neq \phi$, then the class:

$$
F(I)=\{M \subset X: M=X \backslash A \text { for some } A \in I\}
$$

is a filter on $X$, called the filter associated with $I$.

\section{Definition 2.9}

A non trivial ideal $I$ in $X$ is called admissible if $\{x\} \in I$ for each $x \in X$.

\section{Note 2.1}

If $I$ is an admissible ideal, then usual convergence in $X$ implies $I$ convergence in $X$.

\section{Remark 2.2}

If $I$ is an admissible ideal, then usual rough convergence implies rough $I$-convergence.

\section{Definition 2.10}

Let $f$ be a given continuous function defined over the closed interval $[0,1]$. A triple sequence of fuzzy Bernstein-Stancu polynomials $\left(S_{r s t, p, q}(f, X)\right)$ in a metric 
space $(X,|.,|$.$) and \beta$ be a non-negative real number is said to be rough ideal convergent or $\beta I$-convergent to $(f, X)$, denoted by $S_{r s t, p, q}(f, X) \rightarrow^{\beta I}(f, X)$, if for any $\epsilon>0$ we have:

$$
\left\{(r, s, t) \in \mathbb{N}^{3}:\left|S_{r s t, p, q}(f, X)-(f, X)\right| \geq \beta+\epsilon\right\} \in I .
$$

In this case $\left(S_{r s t, p, q}(f, X)\right)$ is called $\beta I$-convergent to $(f, X)$ and a triple sequence of fuzzy Bernstein-Stancu polynomials $\left(S_{r s t, p, q}(f, X)\right)$ is called rough I-convergent to $(f, X)$ with $\beta$ as roughness of degree. If $\beta=0$ then it is ordinary $I$-convergent.

\section{Note 2.2}

In general, let $f$ be a given continuous function defined over the closed interval $[0,1]$. A triple sequence of fuzzy Bernstein-Stancu polynomials $\left(S_{r s t, p, q}(g, X)\right)$ is not $I$-convergent in usual sense and $\mid S_{r s t, p, q}(f, X)-S_{r s t, p, q}$ $(g, X) \mid \leq \beta$ for all $(r, s, t) \in \mathbb{N}^{3}$ or:

$$
\left\{(r, s, t) \in \mathbb{N}^{3}:\left|S_{r s t, p, q}(f, X)-S_{r s t, p, q}(g, X)\right| \geq \beta\right\} \in I
$$

for some $\beta>0$. Under these conditions, the triple sequence of fuzzy Bernstein-Stancu polynomials $\left(S_{r s t, p, q}\right.$ $(f, X))$ is $\beta I$-convergent.

\section{Note 2.3}

It is obvious that it is not necessary $\beta I$-limit of a sequence $S_{r s t, p, q}(f, X)$ of fuzzy Bernstein-Stancu polynomial is unique.

\section{Definition 2.11}

Let us consider $\beta I$-limit set of $(f, X)$, which is denoted by:

$$
I-\operatorname{LIM}^{\beta} S_{r s t, p, q}(f, X)=\left\{f: S_{r s t, p, q}(f, X) \rightarrow^{\beta I}(f, X)\right\},
$$

then the triple sequence of fuzzy Bernstein-Stancu polynomials $\left(S_{r s t, p, q}(f, X)\right)$ is said to be $\beta I$-convergent if $I$ $\operatorname{LIM}^{\beta} S_{r s t, p, q}(f, X) \neq \phi$ and $\beta$ is called a rough $I-$ convergence degree of $S_{r s t, p, q}(f, X)$.

\section{Definition 2.12}

Let $f$ be a given continuous function defined on the closed interval $[0,1]$. A triple sequence of fuzzy Bernstein-Stancu polynomials $\left(S_{r s t, p, q}(f, X)\right)$ is said to be $I$-analytic if there exists a positive real number $M$ such that:

$$
\left\{(r, s, t) \in \mathbb{N}^{3}:\left|S_{r s t, p, q}(f, X)\right|^{1 / m+n+k} \geq M\right\} \in I .
$$

\section{Definition 2.13}

Let $f$ be a given continuous function defined over the closed interval $[0,1]$. A point $L \in X$ is said to be an $I$ accumulation point of a fuzzy Bernstein-Stancu polynomials $\left(S_{r s t, p, q}(f, X)\right)$ in a given space having the metric $(X, d)$ iff for each $\_>0$ the set:

$$
\left\{\begin{array}{l}
(r, s, t) \in \mathbb{N}^{3}: d\left(S_{r s t, p, q}(f, X),(f, X)\right) \\
=\left|S_{r s t, p, q}(f, X)-(f, X)\right|<\epsilon
\end{array}\right\} \notin I .
$$

We denote the set of all $I$-accumulation points of $\left(S_{r s t, p, q}(f, X)\right)$ by I $\left.\left(\Gamma\left(S_{r s t, p, q}(f, X)\right)\right)\right)$.

\section{Definition 2.14}

Let $f$ be a given continuous function defined over the closed interval $[0,1]$. A given triple sequence of fuzzy Bernstein-Stancu polynomials $\left(S_{r s t, p, q}(f, X)\right)$ is said to be rough $I$-convergent if $I-\operatorname{LIM}^{\beta} S_{r s t, p, q}(f, X) \neq$ $\phi$. It is clear that if $I$-LIM ${ }^{\beta} S_{r s t, p, q}(f, X) \neq \phi$ for a triple sequence of fuzzy Bernstein-Stancu polynomials $\left(S_{r s t, p, q}(f, X)\right)$ of real numbers, then we have:

$$
\begin{aligned}
& I-\operatorname{LIM}^{\beta} S_{r s t, p, q}(f, X) \\
& =\left[I-\lim \sup S_{r s t, p, q}(f, X)-\beta, I-\liminf S_{r s t, p, q}(f, X)+\beta\right] .
\end{aligned}
$$

\section{Definition 2.15}

Let $f$ be a given continuous function defined over the closed interval $[0,1]$. The number of $\bar{\beta}=\{\beta \geq 0$ : I$\left.\operatorname{LIM}^{\beta} S_{r s t, p, q}(f, X) \neq \phi\right\}$ is said to be rough $I$-core $S_{r s t, p, q}$ $(f, X)$ of triple sequence of fuzzy Bernstein-Stancu polynomials $\left(S_{r s t, p, q}(f, X)\right)$.

\section{Main Results}

\section{Theorem 3.1}

Let $f$ be a given continuous function defined over the closed interval $[0,1]$. A triple sequence of fuzzy Bernstein-Stancu polynomials $\left(S_{r s t, p, q}(f, X)\right)$ of real numbers and $I \subset 2^{\mathbb{N}}$ be an admissible ideal, we obtain $\operatorname{diam}\left(\mathrm{I}_{-} \mathrm{LIM}^{\beta} S_{r s t, p, q}(f, X)\right) \leq 2 \beta$. In general, diam $\left(I-\mathrm{LIM}^{\beta}\right.$ $\left.S_{r s t, p, q}(f, X)\right)$ has got an upper bound.

\section{Proof} $(f, X)$ :

Assume that diam $\left(\operatorname{LIM}^{\beta} S_{r s t, p, q}(f, X)\right)$. Then, $\exists S_{r s t, p, q}$

$$
\begin{aligned}
& S_{r s t, p, q}(q, X) \in \operatorname{LIM}^{\beta} S_{r s t, p, q}(f, X) \\
& \ni:\left|S_{r s t, p, q}(p, X)-S_{r s t, p, q}(q, X)\right|>2 \beta .
\end{aligned}
$$


Take $\quad \epsilon \in\left(0, \frac{\left|S_{r s t, p, q}(p, X)-S_{r s t, p, q}(q, X)\right|}{2}-\beta\right)$.

Because:

$$
S_{r s t, p, q}(p, X), S_{r s t, p, q}(q, X) \in I-\operatorname{LIM}^{\beta} S_{r s t, p, q}(f, X),
$$

we have $A_{1}(\epsilon) \in I$ and $A_{2}(\epsilon) \in I$ for each $\epsilon>0$, in which:

$$
A_{1}(\epsilon)=\left\{(u, v, w) \in \mathbb{N}^{3}:\left|S_{r s t, p, q}(f, X)-S_{r s t, p, q}(p, X)\right| \geq r+\epsilon\right\}
$$

and:

$$
A_{2}(\epsilon)=\left\{(u, v, w) \in \mathbb{N}^{3}:\left|S_{r s t, p, q}(f, X)-S_{r s t, p, q}(q, X)\right| \geq r+\epsilon\right\} .
$$

By utilizing the properties $F(I)$, we obtain:

$$
\left(A_{1}(\epsilon)^{c} \cap A_{2}(\epsilon)^{c}\right) \in F(I)
$$

Therefore we can write:

$$
\begin{aligned}
& \left|S_{r s t, p, q}(p, X)-S_{r s t, p, q}(q, X)\right| \leq\left|S_{r s t, p, q}(f, X)-S_{r s t, p, q}(p, X)\right| \\
& +\left|S_{r s t, p, q}(f, X)-S_{r s t, p, q}(q, X)\right|<(\beta+\epsilon)+(\beta+\epsilon)<2(\beta+\epsilon),
\end{aligned}
$$

for all $(r, s, t) \in A_{1}(\epsilon)^{c} \cap A_{2}(\epsilon)^{c}$ which is a contradiction. Hence diam $\left(\operatorname{LIM}^{\beta} S_{r s t, p, q}(f, X) \leq 2 \beta\right.$.

Now, it is time to take into consideration a triple sequence of fuzzy Bernstein-Stancu polynomials of $\left(S_{r s t, p, q}(f, X)\right)$ of real numbers such that $I-\lim _{r s t \rightarrow \infty} S_{r s t, p, q}$ $(f, X)=(f, X)$.

Let $\epsilon>0$. Then we can write:

$$
\left\{(r, s, t) \in \mathbb{N}^{3}:\left|S_{r s t, p, q}(f, X)-(f, X)\right| \geq \epsilon\right\} \in I
$$

Therefore we have:

$$
\begin{aligned}
& \left|S_{r s t, p, q}(f, X)-S_{r s t, p, q}(p, X)\right| \leq\left|S_{r s t, p, q}(f, X)-(f, X)\right| \\
& +\left|(f, X)-S_{r s t, p, q}(p, X)\right| \\
& \leq\left|S_{r s t, p, q}(f, X)-(f, X)\right|+\beta \leq \beta+\epsilon,
\end{aligned}
$$

for each:

$$
\begin{aligned}
& S_{r s t, p, q}(p, X) \in \bar{S}_{\beta}((f, X)): \\
& =\left\{S_{r s t, p q}(p, X) \in \mathbb{R}^{3}:\left|S_{r s t, p, q}(p, X)-(f, X)\right| \geq \beta\right\} .
\end{aligned}
$$

Next we obtain:

$$
\left|S_{r s t, p, q}(f, X)-S_{r s t, p, q}(p, X)\right|<\beta+\epsilon
$$

for each $(r, s, t) \in\left\{(r, s, t) \in \mathbb{N}^{3}: \mid S_{r s t, p, q}(f, X)-(f, X)<\epsilon\right\}$. Because the triple sequence of fuzzy Bernstein-Stancu polynomials of $S_{r s t, p, q}(f, X)$ is I-convergent to $(f, X)$, we have:

$$
\left\{(r, s, t) \in \mathbb{N}^{3}:\left|S_{r s t, p, q}(f, X)-(f, X)\right|<\epsilon\right\} \in F(I) .
$$

Therefore, we obtain $p \in I$-LIM ${ }^{r} \quad S_{r s t, p, q} \quad(f, X)$. Consequently, we can write:

$$
I-\operatorname{LIM}^{\beta} S_{r s t, p, q}(f, X)=\bar{S}_{\beta}((f, X)) .
$$

Since diam $\left(\bar{S}_{\beta}((f, X))\right)=2 \beta$, this shows that in general, the upper bound $2 \beta$ of the diameter of the set $I$ $\operatorname{LIM}^{\beta} S_{r s t, p, q}(f, X)$ is not lower bound.

\section{Theorem 3.2}

Let $f$ be a given continuous function defined over the closed interval $[0,1]$. A triple sequence of fuzzy Bernstein-Stancu polynomials $\left(S_{r s t, p, q}(f, X)\right)$ of real numbers, $I \subset 3^{\mathbb{N}}$ be an admissible ideal. For an arbitrary $(f, c) \in I\left(\Gamma_{X}\right)$, we obtain $\left|S_{r s t, p, q}(f, X)-(f, c)\right| \leq \beta$ for all $S_{r s t, p, q}(f, X) \in I-\operatorname{LIM}^{\beta} S_{r s t, p, q}(f, X)$.

\section{Proof}

Assume to the contrary that there is a point $(f, c) \in I\left(\Gamma_{X}\right)$ and $S_{r s t, p, q}(f, X) \in I-\operatorname{LIM}^{\beta} S_{r s t, p, q}(f, X)$ such that | $\quad \begin{array}{lll}S_{r s t, p, q} & (f, X)-(f, c) \mid>\beta . & \text { Define }\end{array}$ $\epsilon:=\frac{\left|S_{r s t, p, q}(f, X)-(f, c)\right|-\beta}{3}$. Then:

$\left\{(r, s, t) \in \mathbb{N}^{3}:\left|S_{r s t, p, q}(f, X)-(f, c)\right|<\epsilon\right\}$

$\subseteq\left\{(r, s, t) \in \mathbb{N}^{3}:\left|S_{r s t, p, q}(f, X)-(f, X)\right| \geq \beta+\epsilon\right\}$.

Due to the fact that $(f, c) c \in I\left(\Gamma_{X}\right)$, we have:

$$
\left\{(r, s, t) \in \mathbb{N}^{3}:\left|S_{r s t, p, q}(f, X)-(f, c)\right|<\epsilon\right\} \notin I .
$$

However, using the definition of I-convergence, because:

$$
\left\{(r, s, t) \in \mathbb{N}^{3}:\left|S_{r s t, p, q}(f, X)-(f, X)\right| \geq \beta+\epsilon\right\} \in I,
$$

so by (3.2) we have:

$$
\left\{(r, s, t) \in \mathbb{N}^{3}:\left|S_{r s t, p, q}(f, X)-(f, c)\right|<\epsilon\right\} \in I,
$$

which is a contradiction to the fact $(f, c) \in I\left(\Gamma_{X}\right)$. On the other hand, if $(f, c) \in I\left(\Gamma_{X}\right)$ i.e.: 


$$
\left\{(r, s, t) \in \mathbb{N}^{3}:\left|S_{r s t, p, q}(f, X)-(f, c)\right|<\epsilon\right\} \notin I,
$$

then:

$$
\left\{(r, s, t) \in \mathbb{N}^{3}:\left|S_{r s t, p, q}(f, X)-(f, X)\right| \geq \beta+\epsilon\right\} \notin I,
$$

which is a contradiction to the fact that $(f, X) \in I$-LIM ${ }^{\beta}$ $S_{r s t, p, q}(f, X)$.

\section{Theorem 3.3}

Let $f$ be a continuous function defined on the closed interval $[0,1]$. A triple sequence of fuzzy BernsteinStancu polynomials:

$$
\begin{aligned}
& \left(S_{r s t, p, q}(f, X)\right) \rightarrow^{I}(f, X) \\
& \Leftrightarrow I-\operatorname{LIM}^{\beta} S_{r s t, p, q}(f, X)=\bar{S}_{\beta}((f, X)) .
\end{aligned}
$$

\section{Proof}

Necessity: By Theorem 3.1.

\section{Sufficiency}

Assume that $I$-LIM ${ }^{\beta} S_{r s t, p, q}(f, X)=\bar{S}_{\beta}((f, X))(\neq \phi)$. Thus the triple sequence spaces of fuzzy BernsteinStancu polynomials of $\left(S_{r s t, p, q}(f, X)\right)$ is $I$-analytic. If we assume that $(f, X)$ has another $I$-cluster point $\left(f^{\prime}, X\right)$ different from $(f, X)$. Then the following point:

$$
\begin{aligned}
& (\bar{f}, X)=(f, X)+\frac{\beta}{\left|(f, X)-\left(f^{\prime}, X\right)\right|}\left((f, X)-\left(f^{\prime}, X\right)\right) \\
& (\bar{f}, X)-\left(f^{\prime}, X\right)=(f, X)-\left(f^{\prime}, X\right) \\
& +\frac{\beta}{\left|(f, X)-\left(f^{\prime}, X\right)\right|}\left[\left((f, X)-\left(f^{\prime}, X\right)\right)-\left(\left(f^{\prime}, X\right)-\left(f^{\prime}, X\right)\right)\right] \\
& \left|(\bar{f}, X)-\left(f^{\prime}, X\right)\right|=\left|(f, X)-\left(f^{\prime}, X\right)\right| \\
& +\frac{\beta}{\left|(f, X)-\left(f^{\prime}, X\right)\right|}\left|(f, X)-\left(f^{\prime}, X\right)\right| \\
& \left|(\bar{f}, X)-\left(f^{\prime}, X\right)\right|=\left|(f, X)-\left(f^{\prime}, X\right)\right|+\beta>\beta .
\end{aligned}
$$

Since $\left(f^{\prime}, X\right) \in I\left(\Gamma_{X}\right)$, using Theorem 3.2, $(\bar{f}, X) \notin I-$ $\operatorname{LIM}^{\beta} S_{r s t, p, q}(f, X)$. It is not possible as $|(\bar{f}, X)-(f, X)|=\beta$ and $I$-LIM ${ }^{r} S_{r s t, p q}(f, X)=\bar{S}_{\beta}((f, X))$. Since $(f, X)$ is the unique- $I$ cluster point of $(f, X)$. Thus $\Rightarrow S_{\text {rst.p,q }}(f, X) \rightarrow^{I}(f, X)$.

\section{Corollary 3.1}

If $(X,|.|$,$) is a strictly convex spaces and at the same$ time $f$ is a given continuous function given over the closed interval $[0,1]$. A triple sequence of fuzzy Bernstein-Stancu polynomials $\left(S_{r s t, p, q}(f, X)\right) \in X$, there exists $y_{1}, y_{2} \in I-\operatorname{LIM}^{\beta} S_{r s t, p, q}(f, X)$ such that $\left|y_{1}-y_{2}\right|=2 \beta$, then this triple sequence of fuzzy $(f, X) \rightarrow^{I} \frac{y_{1}+y_{2}}{2}$.

\section{Proof}

Omitted.

\section{Theorem 3.4}

If $I-\mathrm{LIM}^{\beta} \neq \phi$, then $I$-lim sup $S_{r s t, p, q}(f, X)$ and $I$-lim inf $S_{r s t, p, q}(f, X)$ are in the set $I$-LIM ${ }^{2 \beta} S_{r s t, p, q}(f, X)$.

\section{Proof}

We know that $I$-LIM ${ }^{\beta} S_{r s t, p, q}(f, X) \neq \phi$, a triple sequence of fuzzy Bernstein-Stancu polynomials of $\left(S_{r s t, p, q}(f, X)\right)$ is $I$-analytic. The number $I$-lim inf $S_{r s t, p, q}$ $(f, X)$ is an $I$-cluster point of $(f, X)$ and thus, we obtain:

$$
\begin{aligned}
& \left|(f, X)-I-\liminf S_{r s t, p, q}(f, X)\right| \\
& \leq \beta \forall(f, X) \in I-\operatorname{LIM}^{\beta}(f, X) .
\end{aligned}
$$

Let $A=\left\{(r, s, t) \in \mathbb{N}^{3}:\left|(f, X)-S_{r s t, p, q}(f, X)\right| \geq \beta+\epsilon\right\}$. Now if $(r, s, t) \notin A$, then:

$$
\begin{aligned}
& \left|S_{r s t, p, q}(f, X)-\left(I-\liminf S_{r s t, p, q}(f, X)\right)\right| \leq\left|S_{r s t, p, q}(f, X)-(f, X)\right| \\
& +\left|(f, X)-\left(I-\liminf S_{r s t, p, q}(f, X)\right)\right|<2 \beta+\epsilon .
\end{aligned}
$$

Thus:

$$
I-\liminf f S_{r s t, p, q}(f, X) \in I-L I M^{2 \beta} S_{r s t, p, q}(f, X) .
$$

In a similar way, it can be shown that $I$-lim sup $X_{m n k} \in I$-LIM ${ }^{2 \beta} X_{m n k}$.

\section{Corollary 3.2}

Let $f$ be a given continuous function defined on the closed interval $[0,1]$. A triple sequence of fuzzy Bernstein-Stancu polynomials $\left(S_{r s t, p, q}(f, X)\right)$ of real numbers, if $I-\operatorname{LIM}^{\beta} S_{r s t, p, q}(f, X) \neq \phi$, then:

$$
I-\operatorname{core}\{(f, X)\} \subseteq I-L I M^{2 \beta} S_{r s t, p, q}(f, X) .
$$

\section{Proof}

We obtain:

$$
\begin{aligned}
& I-\operatorname{LIM}^{\beta} S_{r s t, p q}(f, X) \\
& =\left[I-\limsup S_{r s t, p, q}(f, X)-2 \beta, I-\liminf S_{r s t, p, q}(f, X)+2 \beta\right] .
\end{aligned}
$$

Under these conditions, the result yield in from Theorem 3.4. 


\section{Theorem 3.5}

Let $f$ be a given continuous function defined over the closed interval $[0,1]$. A triple sequence of fuzzy Bernstein-Stancu polynomials $\left(S_{r s t, p, q}(f, X)\right)$ of real numbers. Then the diam (I-core $\left.\left\{S_{r s t, p, q}(f, X)\right\}\right)$ of the set:

$$
\begin{aligned}
& I-\operatorname{core}\left\{S_{r s t, p, q}(f, X)\right\} \\
& =\beta \Leftrightarrow I-\operatorname{core}\{(f, X)\}=I-\operatorname{LIM}^{\beta} S_{r s t, p, q}(f, X)
\end{aligned}
$$

Proof:

$$
\begin{aligned}
& \operatorname{diam}\left(I-\operatorname{core}\left\{S_{r s t, p, q}(f, X)\right\}\right)=\beta \\
& \Leftrightarrow\left(I-\limsup S_{r s t, p, q}(f, X)\right)-\left(I-\limsup X_{m n k}\right)=\beta \\
& \Leftrightarrow I-\operatorname{core}\left\{X_{m n k}\right\}=\left[I-\liminf X_{m n k}, I-\limsup S_{r s t, p, q}(f, X)\right] \\
& =\left[I-I-\limsup S_{r s t, p, q}(f, X)-\beta, I-\liminf S_{r s t, p, q}(f, X)+r\right] \\
& =I-\operatorname{LIM}^{\beta} S_{r s t, p, q}(f, X) .
\end{aligned}
$$

Also it is easy to see that:

(i) $\beta>\operatorname{diam}\left(I\right.$-core $\left.\left\{S_{r s t, p, q}(f, X)\right\}\right) \Leftrightarrow$-core $\left\{S_{r s t, p, q}\right.$ $(f, X)\} \subset I-\operatorname{LIM}^{\beta} S_{r s t, p, q}(f, X)$,

(ii) $\beta>\operatorname{diam}\left(I\right.$-core $\left.\left\{S_{r s t, p, q}(f, X)\right\}\right) \Leftrightarrow I-\operatorname{LIM}^{\beta} S_{r s t, p, q}(f, X)$ $\subset I$-core $\left\{S_{r s t, p, q}(f, X)\right\}$

\section{Theorem 3.6}

Let $f$ be a continuous function defined over the closed interval $[0,1]$. A triple sequence of fuzzy BernsteinStancu polynomials $\left(S_{r s t, p, q}(f, X)\right)$ of real numbers, if $\bar{\beta}=$ $\inf \left\{\beta \geq 0: I-\operatorname{LIM}^{\beta} S_{r s t, p, q}(f, X) \neq \phi\right.$, then $\bar{\beta}=$ radius $(I-$ core $\left.\left\{S_{r s t, p, q}(f, X)\right\}\right)$.

\section{Proof}

If the set I-core $\left\{S_{r s t, p, q}(f, X)\right\}$ is singleton, then $\operatorname{radius}\left(I-\operatorname{core}\left\{S_{r s t, p, q}(f, X)\right\}\right)=0$ and the triple sequence of fuzzy Bernstein-Stancu polynomials is I-convergent, i.e., $I$-LIM $S_{r s t, p, q}(f, X) \neq \phi$. Hence we get $\bar{\beta}=$ radius (I-core $\left.\left\{S_{r s t, p, q}(f, X)\right\}\right)=0$.

Now assume that the set I-core $\left\{S_{r s t, p, q}(f, X)\right\}$ is not a singleton. We may write $I$-core $\left\{S_{r s t, p, q}(f, X)\right\}=[a$, $b$ ], where $a=I$-lim inf $S_{r s t, p, q}(f, X)$ and $b=I$-lim sup $S_{r s t, p, q}(f, X)$.

Now assume that $\bar{\beta} \neq$ radius $\left(I\right.$-core $\left.\left\{S_{r s t, p, q}(f, X)\right\}\right)$.

If $\bar{\beta}<$ radius (I-core $\left\{X_{m n k}\right\}$ ), then define $\bar{\epsilon}=\frac{\frac{b-a}{2}-\bar{\beta}}{3}$. Now, be definition of $\bar{\beta}$ implies that $I-\operatorname{LIM}^{\bar{\beta}+\bar{\epsilon}} S_{r s t, p, q}(f, X) \neq \phi$, given $\epsilon>0$ :

$$
\exists l \in \mathbb{R}: A\left\{\begin{array}{l}
(r, s, t) \in \mathbb{N}: \mid S_{r s t, p, q}(f, X) \\
-(f, X) \mid \geq(\bar{\beta}+\bar{\epsilon})+\epsilon
\end{array}\right\} \in I .
$$

Since $\bar{\beta}+\bar{\epsilon}<\frac{b-a}{2}$ which is a contradiction of the definition of $a$ and $b$.

If $\bar{\beta}>$ radius (I-core $\left.\left\{S_{r s t, p, q}(f, X)\right\}\right)$, then define $\bar{\epsilon}-\frac{\bar{\beta}-\frac{b-a}{2}}{3}$ and $\beta^{\prime}=\bar{\beta}-2 \bar{\epsilon}$.

It is clear that $0 \leq \beta^{\prime} \leq \bar{\beta}$ and using the descriptions of a and $\mathrm{b}$, the number $\frac{b-a}{2} \in I-L I M \beta^{\prime} S_{r s t, p, q}(f, X)$. Under these conditions, we obtaint:

$$
\bar{\beta} \in\left\{\beta \geq 0: I-\operatorname{LIM}^{\beta} S_{r s t, p, q}(f, X) \neq \phi\right\},
$$

which is a contradiction to the equality:

$$
\bar{\beta}=\inf \left\{\beta \geq 0: I-\operatorname{LIM}^{\beta} S_{r s t, p, q}(f, X) \neq \phi\right\} \text { as } \beta^{\prime}<\beta .
$$

\section{Corollary 3.3}

Let $f$ be a continuous function defined on the closed interval $[0,1]$. A triple sequence of fuzzy BernsteinStancu polynomials $\left(S_{r s t, p, q}(f, X)\right)$ of real numbers, then $I$ core $\left\{S_{r s t, p q}(f, X)\right\}=I-\operatorname{LIM}^{2 \bar{\beta}} S_{r s t, p, q}(f, X)$.

\section{Proof}

It follows that Theorem 3.5 and Theorem 3.6.

\section{Example 3.1}

Let $f$ be a continuous function defined on the closed interval $[0,1]$.

With the help of Matlab, we show comparisons and some illustrative figures on the convergence of operators given in (1.2) to the function $f(X)=1+X^{3} \sin (14 X)$ under different arguments.

From Fig. 1a, we can see that as the value the $q$ and $p$ goes to 1 under the conditions $0<q<p \leq 1,(p, q)$ Bernstein-Stancu operators given by (1.2) converges to the function $f(X)=1+X^{3} \sin (14 X)$. From these figures, namely 1 (a) and (b), we can see that for $\eta=\mu=0$, when the values of the rst go up, $(p, q)$-Bernstein-Stancu operators given by (1.2) converges to the function. In a similar manner, using the Fig. 2a, we may conclude that for $\eta=\mu=5$, when the values of the $q$ and $p$ go to 1 or else the condition $0<q<p \leq 1$ is provided, $(p, q)$ Bernstein-Stancu operators given by (1.2) converges towards the function. Using the Fig. 2a and $2 b$, we can conclude that as the values of the $[r s t]$ go up, $(p, q)$ Bernstein-Stancu operators given by $f(X)=1+X^{3}$ $\sin (14 X)$ converges to the function. 


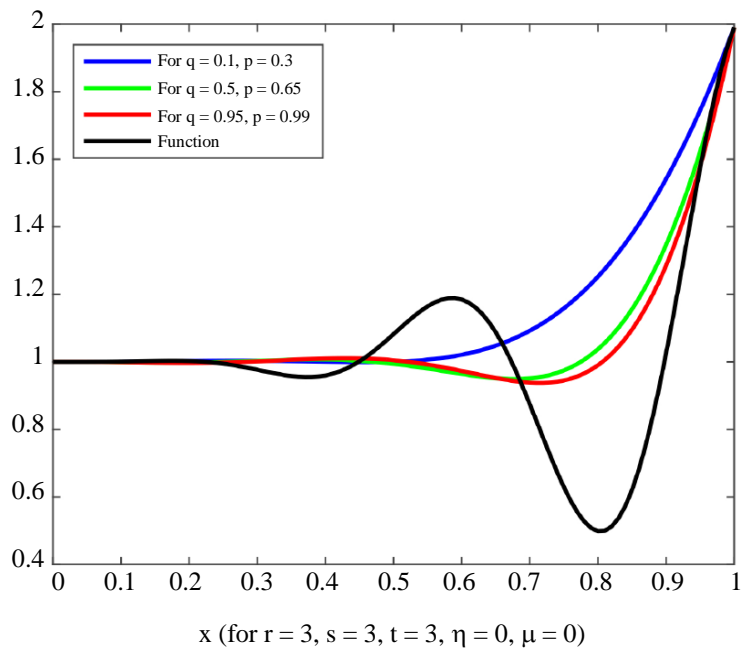

(a)

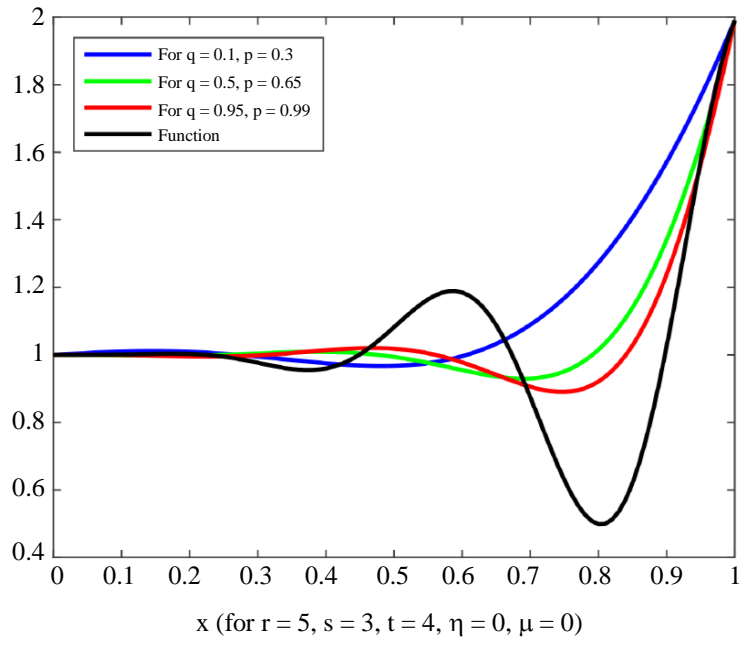

(b)

Fig. 1: $(p, q)$-Bernstein-Stancu operators

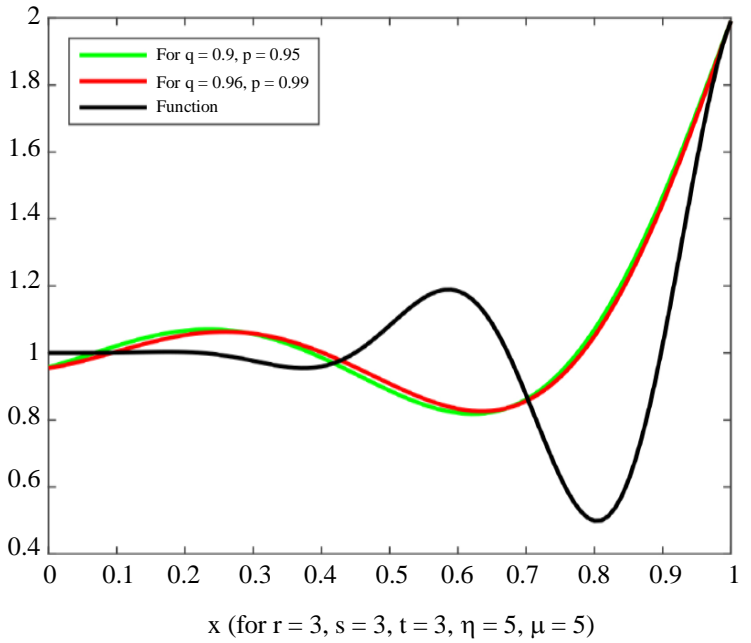

(a)

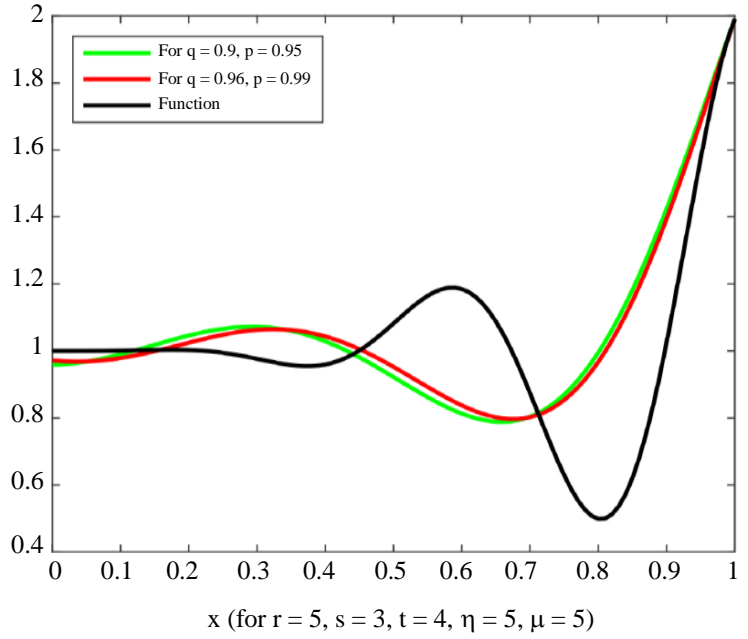

(b)

Fig. 2: $(p, q)$-Bernstein-Stancu operators

\section{Conclusion}

We introduced and studied some basic properties of fuzzy Bernstein-Stancu operators of rough Iconvergent of triple sequences and also studied the set of all fuzzy Bernstein-Stancu operators of rough Ilimits of a triple sequence and relation between analytic ness and Fuzzy Bernstein-Stancu operators of rough I-core of a triple sequence.

For the reference sections, considered the following introduction described the main results are motivating the research.

\section{Acknowledgment}

The authors thanks to referees for the comments those improved the presentation of the article.

\section{Author's Contributions}

The authors contributed equally and significantly in writing this paper. All authors read and approved the final manuscript.

\section{Competing Interests}

The authors of the present manuscript declare that there is not any conflict of interests regarding the publication of this study.

\section{References}

Aytar, S., 2008a. Rough statistical convergence. Numer. Funct. Anal. Optim., 29: 291-303. DOI: $10.1080 / 01630560802001064$ 
Aytar, S., 2008b. The rough limit set and the core of a real sequence. Numer. Funct. Anal. Optim., 29: 283-290. DOI: 10.1080/01630560802001056

Esi, A., 2014. On some triple almost lacunary sequence spaces defined by Orlicz functions. Res. Reviews: Discrete Math. Struct., 1: 16-25.

Esi, A. and M.N. Catalbas, 2014. Almost convergence of triple sequences. Global J. Math. Anal., 2: 6-10. DOI: 10.14419/gjma.v2i1.1709

Esi, A. and E. Savas, 2015. On lacunary statistically convergent triple sequences in probabilistic normed space. Applied Math. Inform. Sci., 9: 2529-2534.

Esi, A., S. Araci and M. Acikgoz, 2016. Statistical convergence of Bernstein operators. Applied Math. Inform. Sci., 10: 2083-2086. DOI: $10.18576 /$ amis/100610

Dutta, A.J., A. Esi and B.C. Tripathy, 2013. Statistically convergent triple sequence spaces defined by Orlicz function. J. Math. Anal., 4: 16-22.

Debnath, S., B. Sarma and B.C. Das, 2015. Some generalized triple sequence spaces of real numbers. J. Nonlinear Anal. Optim., 6: 71-79.

Dundar, E. and C. Cakan, 2014. Rough I-convergence. Demonstr. Math., 47: 638-651.

DOI: 10.2478/dema-2014-0051
Khan, K. and D.K. Lobiyal, 2015. Bèzier curves based on Lupaş $(p, q)$-analogue of Bernstein polynomials in CAGD. arXiv: 1505.01810[cs.GR].

Phu, H.X., 2001. Rough convergence in normed linear spaces. Numer. Funct. Anal. Optim., 22: 199-222. DOI: $10.1081 /$ NFA-100103794

Phu, H.X., 2002. Rough continuity of linear operators. Numer. Funct. Anal. Optim., 23: 139-146. DOI: $10.1081 /$ NFA-120003675

Phu, H.X., 2003. Rough convergence in infinite dimensional normed spaces. Numer. Funct. Anal. Optim., 24: 285-301. DOI: $10.1081 /$ NFA-120022923

Sahiner, A., M. Gurdal and F.K. Duden, 2007. Triple sequences and their statistical convergence. Selcuk J. Applied Math., 8: 49-55.

Sahiner, A. and B.C. Tripathy, 2008. Some I-related properties of triple sequences. Selcuk J. Applied Math., 9: 9-18.

Subramanian, N. and A. Esi, 2015. The generalized tripled difference of $\chi^{3}$ sequence spaces. Global $\mathrm{J}$. Math. Anal., 3: 54-60.

DOI: $10.14419 /$ gjma.v3i2.4412 